\title{
Estrategias de prevención neuromuscular para las lesiones de ligamento cruzado anterior sin contacto en jugadoras de baloncesto. Revisión narrativa
}

Gómez-Tomás, Cinta; Rial Rebullido, Tamara; Chulvi-Medrano, Iván

Estrategias de prevención neuromuscular para las lesiones de ligamento cruzado anterior sin contacto en jugadoras de baloncesto. Revisión narrativa

MHSalud, vol. 18, núm. 2, 2021

Universidad Nacional, Costa Rica

Disponible en: https://www.redalyc.org/articulo.oa?id=237066090007 DOI: https://doi.org/10.15359/mhs.18-2.7

\section{(c) (1) (9)}

Esta obra está bajo una Licencia Creative Commons Atribución-NoComercial-SinDerivar 3.0 Internacional. 


\title{
Estrategias de prevención neuromuscular para las lesiones de ligamento cruzado anterior sin contacto en jugadoras de baloncesto. Revisión narrativa
}

\author{
Neuromuscular Prevention Strategies for The Non-Contact Anterior Cruciate Ligament Injury in Female \\ Basketball Players. Narrative Review \\ Estratégias de prevenção neuromuscular para lesões do ligamento cruzado anterior sem contato em jogadoras de \\ basquete. Revisão narrativa \\ Cinta Gómez-Tomás \\ Universidad Católica San Antonio de Murcia, España \\ cgomez@ucam.edu \\ DOI: https://doi.org/10.15359/mhs.18-2.7 \\ Redalyc: https://www.redalyc.org/articulo.oa?
}

(iD) https://orcid.org/0000-0002-3288-3253

Tamara Rial Rebullido

University of Pennsylvania, Perelman's School of Medicine

Department of Orthopeadic Surgery, Estados Unidos

rialtamara@gmail.com

iD https://orcid.org/ 0000-0001-8526-6323

Iván Chulvi-Medrano

Universidad de Valencia, España

ivanchulvimedrano@gmail.com

(iD https://orcid.org/0000-0002-6612-1759

Recepción: 24 Junio 2020

Aprobación: 12 Febrero 2021

\section{RESUMEN:}

Introducción: La lesión del ligamento cruzado anterior sin contacto tiene una mayor incidencia en las mujeres deportistas en edad adolescente. Las características propias del baloncesto (el salto, el aterrizaje o los cambios de dirección que se producen cada 2.0 a 2.82 segundos) junto con las características biomecánicas y hormonales propias de la mujer (mayor valgo de rodillas, laxitud articular y ciclo menstrual) hace que nos encontremos ante un sector poblacional de especial interés para implementar programas de prevención neuromuscular. El objetivo de este estudio ha sido realizar una revisión bibliográfica para proponer un programa de prevención neuromuscular para las lesiones de ligamento cruzado anterior en mujeres jugadoras de baloncesto. Material y método: La búsqueda se realizó en las bases de datos PubMed, SportDiscus y PEDro entre diciembre 2019-enero 2020. Los descriptores "anterior cruciate ligament", "neuromuscular training", "injury rates", "basketball" y "women" se utilizaron como criterio de búsqueda. Resultados: Se analizaron 20 artículos que fueron seleccionados acorde a los criterios de inclusión: estudios que aporten información sobre factores de riesgo y programas de prevención de la lesión de ligamento cruzado anterior en mujeres sanas, atletas/deportistas. Se clasificó la información obtenida según estrategia o información preventiva aportada. Conclusiones: Un programa de prevención neuromuscular para lesiones de ligamento cruzado anterior sin contacto en mujeres jugadoras de baloncesto debe basarse en cuatro aspectos fundamentales: entrenamiento de fuerza de miembro inferior, pliometría, retroalimentación en el aterrizaje y trabajo central de tronco.

PALABRAS CLAVE: mujer, lesión, baloncesto, prevención, ligamento cruzado anterior.

\section{Abstract:}

Introducction: Non-contact anterior cruciate ligament injury has a higher incidence in adolescent female athletes. The characteristics of basketball (jumping and landing) together with the biomechanical and hormonal characteristics of women (greater valgus at the knees, joint laxity and menstrual cycle) make us find ourselves before a population sector of special interest to implement neuromuscular prevention programs. The objective of this study has been a literature review to propose neuromuscular prevention programs for non-contact anterior cruciate ligament injury in female basketball players. Material and method: The search was performed in the PubMed, SportDiscus and PEDro databases between December 2019-January 2020. The keywords "anterior cruciate ligament", "neuromuscular training", "injury rates", "basketball" and "women" were used as search criteria. 
Results: 20 articles that were selected according to the inclusion criteria were analyzed: studies that provide information on risk factors and prevention programs for anterior common ligament injury in healthy women and athletes. The information obtained was classified according to the preventive strategy provided. Conclusions: A neuromuscular prevention program for non-contact anterior cruciate ligament injury in female basketball players should be based on four main aspects: lower limb strength training, plyometrics, feedback on landing and central trunk work.

KEYWORDS: woman, injury, basketball, prevention, anterior cruciate ligament.

\section{Resumo:}

Introdução: A lesão do ligamento cruzado anterior sem contato tem maior incidência em atletas adolescentes do sexo feminino. As características do basquete (salto, aterrissagem ou mudanças de direção que ocorrem a cada 2,0 a 2,82 segundos) juntamente com as características biomecânicas e hormonais da mulher (maior valgo do joelho, frouxidão articular e ciclo menstrual) fazem com que estejamos diante de um setor populacional de especial interesse para implementar programas de prevenção neuromuscular. $\mathrm{O}$ objetivo deste estudo foi realizar uma revisão bibliográfica com o objetivo de propor um programa de prevenção neuromuscular de lesões do ligamento cruzado anterior em jogadoras de basquete do sexo feminino. Material e métodos: A busca foi realizada nas bases de dados PubMed, SportDiscus e PEDro entre dezembro de 2019 e janeiro de 2020. Os descritores "anterior cruciate ligament", "neuromuscular training", "injury rates", "basketball” y "women” foram usados como critérios de pesquisa. Resultados: Foram analisados 20 artigos selecionados de acordo com os critérios de inclusão: estudos que fornecem informações sobre fatores de risco e programas de prevenção da lesão do ligamento cruzado anterior em mulheres saudáveis, atletas / esportistas. As informações obtidas foram classificadas de acordo com a estratégia ou informações preventivas fornecidas. Conclusão: Um programa de prevenção neuromuscular para lesões do LCA sem contato em jogadoras de basquete deve ser baseado em quatro aspectos fundamentais: treinamento de força de membros inferiores, pliometria, retroalimentação na aterrissagem e trabalho central.

PalaVRas-Chave: mulher, lesão, basquete, prevenção, ligamento cruzado anterior.

\section{INTRODUCCIÓN}

El baloncesto es un deporte de contacto que, debido a su naturaleza, frecuencia e intensidad de competición, expone a sus atletas a elevadas fuerzas que pueden resultar potencialmente lesivas (Riva et al., 2016). Acciones deportivas como los cambios de dirección se producen cada 2.0 a 2.82 segundos y se realizan entre 35 y 46 saltos por partido existiendo una gran cantidad de contactos intensos entre quienes juegan (Beynnon et al., 2014). A nivel epidemiológico, según la base de datos de la Asociación Nacional Deportiva Universitaria (NCAA), la mujer registra una mayor tasa de lesiones que implican más de 3 semanas de recuperación (Sanders et al., 2016). En concreto, la incidencia general de lesión de ligamento cruzado anterior sin contacto (en adelante LCA-SC) en adolescentes femeninas es de 0.112 por 1000 exposiciones, frente a 0.063 por mil exposiciones en hombres (Beynnon et al., 2014). Se ha encontrado que las mujeres registran un pico mayor de lesión durante la adolescencia (14-18 años), con 222.7/100.000 personas/año ( $\mathrm{p} / \mathrm{a}$ ) en comparación con 113.2/100.000 p/a para la edad de 19-25 años (Zuckerman et al., 2018).

Los programas de prevención neuromuscular (PNM) para las lesiones de LCA-SC han demostrado ser exitosos en los deportes de alto riesgo (baloncesto, futbol, balonmano...) (Jordan et al., 2017). Las diferencias de movimiento específicas de cada deporte indican la necesidad de adaptar los programas de PNM según la disciplina. Los programas PNM de lesiones de LCA-SC en el baloncesto se deben centrar principalmente en la mejora de la fuerza de las extremidades inferiores y el control neuromuscular del valgo dinámico, principal factor de riesgo biomecánico para la lesión del LCA-SC (Aerts et al, 2015; Caldemeyer et al., 2020; Cherni et al., 2019; Munro et al., 2012; Omi et al., 2018). Después de un programa de PNM de 90 minutos, 3 días a la semana durante 6 semanas, se pueden disminuir los factores de riesgo de lesiones en las extremidades inferiores y mejorar el rendimiento en una muestra compuesta por practicantes de baloncesto, fútbol y voleibol. Por ello resulta importante establecer unas directrices para llevar a cabo un programa de PNM para prevenir las LCA-SC en mujeres deportistas (Myer et al., 2005). 


\section{Mecanismo de lesión en LCA-SC}

En general, la lesión en el LCA-SC se asocia a una carga incorrecta de la rodilla debido a ajustes posturales infructuosos y a cambios rápidos en el entorno (Besier et al., 2001). Particularmente en el caso de las mujeres el mecanismo típico de lesión es causado por los siguientes desequilibrios neuromusculares (Hewett et al., 2010): a) Predominio del ligamento (la mujer presenta una pelvis más ancha y un mayor valgo de rodilla en comparación con los hombres, de modo que el ángulo $Q$ es más elevado y consecuentemente se genera mayor estrés medial sobre los ligamentos de la rodilla); b) Predominio del cuádriceps (debido al menor ratio caudríceps:isquisureales que presenta la mujer, donde se produce una mayor activación del cuádriceps en los movimientos de aterrizaje y de corte, desencadenando una situación de aumento de las fuerzas de cizallamiento anterior de la tibia (Besier et al., 2001; Chappell et al., 2007) y un déficit concurrente de la cadena posterior concretamente en el desarrollo de la fuerza de los músculos isquiosurales (Ahmad et al., 2006); c) Predominio de las piernas (el desequilibrio general dificulta la capacidad de generar fuerzas sinérgicas y estabilizadoras de la rodilla para proteger al LCA-SC) (Pollard et al., 2009) empeorando el adecuado alineamiento de la extremidad inferior, situación que se agrava ante situaciones como la amortiguación tras un salto (figura 1) o un cambio de dirección (acciones muy frecuentes en el baloncesto) o d) Predominio del tronco (empeoramiento del esquema motor, por una mayjan sumberibridadida). mesfular fel fúf flegresettala de este control neuromuscular alterado o disminuido durante la ejecución de movimientos deportivos genera cargas excesivas en las articulaciones de las extremidades inferiores y provoca un aumento del riesgo de lesión del LCA-SC en atletas femeninas (ver figura 1). 
a)

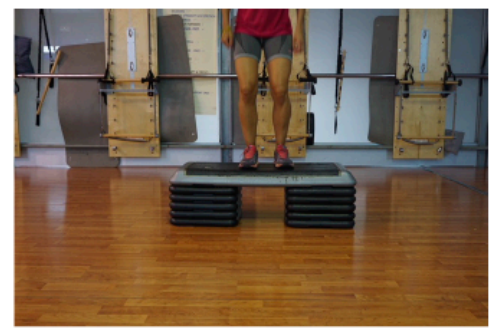

b)

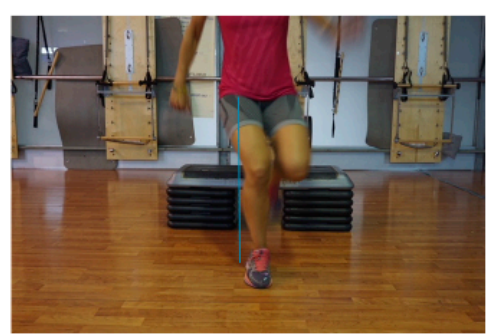

c)

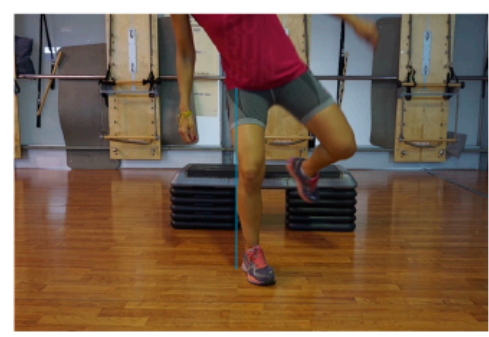

d)

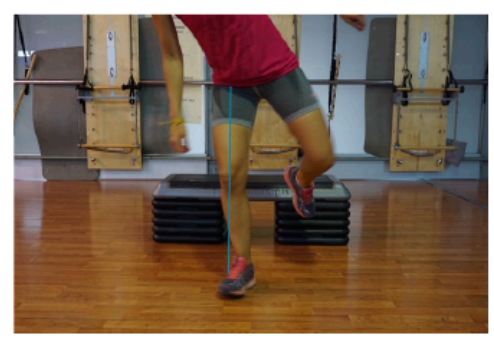

FIGURA 1.

Diferencias entre un aterrizaje monopodal con los aspectos técnicos reforzados (imágenes a y b) y un aterrizaje sin control (imágenes c y d). Reproducido con autorización.

\section{Factores etiológicos}

La etiología LCA-SC es multifactorial, ya que presenta factores de riesgo extrínsecos: i) Competencia en competición versus práctica; ii) Calzado y superficie de juego; iii) Equipo de protección; iv) Condiciones meteorológicas) y factores de riesgo intrínsecos: $\mathrm{v}$ ) Anatómicos (tamaño de la muesca femoral, dimensiones del LCA, grado de laxitud de la rodilla, ángulo Q); vi) neuromusculares; y vii) hormonales (el ciclo menstrual) (Roos et al., 2015).

Por lo tanto, el objetivo del presente estudio fue revisar la evidencia existente en la literatura en relación con las estrategias de PNM para la lesión de LCA-SC en jugadoras de baloncesto y proponer un programa de PNM. 


\section{Justificación}

Al evaluar los programas de PNM para la lesión de LCA-SC, se debe tener en cuenta a qué sector poblacional va dirigido y aplicarlo de manera temprana para incrementar el esquema motor en las fases sensibles de aprendizaje para prevenir lesiones.

\section{Metodología}

Se ha llevado a cabo una búsqueda electrónica de la literatura médica disponible en las bases de datos: PubMed, SportDiscus y PEDro durante los meses de diciembre 2019 hasta enero 2020. La estrategia de búsqueda estuvo basada en las palabras clave: "anterior cruciate ligament", "neuromuscular training", "injury rates", "basketball" y "women", que fueron combinadas con el operador lógico AND. Los criterios de inclusión de los estudios fueron los siguientes: 1) Estudios originales y revisiones publicados sin límite de fecha, 2) en lengua inglesa o castellana, 3) en mujeres sanas y atletas/deportistas, 4) que el principal objetivo fuera el estudio de la prevención de las lesiones del LCA-SC, 5) que aporten información sobre los factores de riesgo de la lesión de LCA-SC, 6) que incluyan un programa de prevención o estrategias de prevención de LCA-SC.

\section{Resultados}

Tras eliminar los duplicados se obtuvieron 655 artículos, y tras la lectura a título y abstract se obtuvo una cantidad de 205 artículos. Una vez se realizó la lectura a texto completo, se obtuvieron 20 artículos para la realización de la síntesis y tratamiento de los datos reportados, como se puede visualizar en la figura 2.

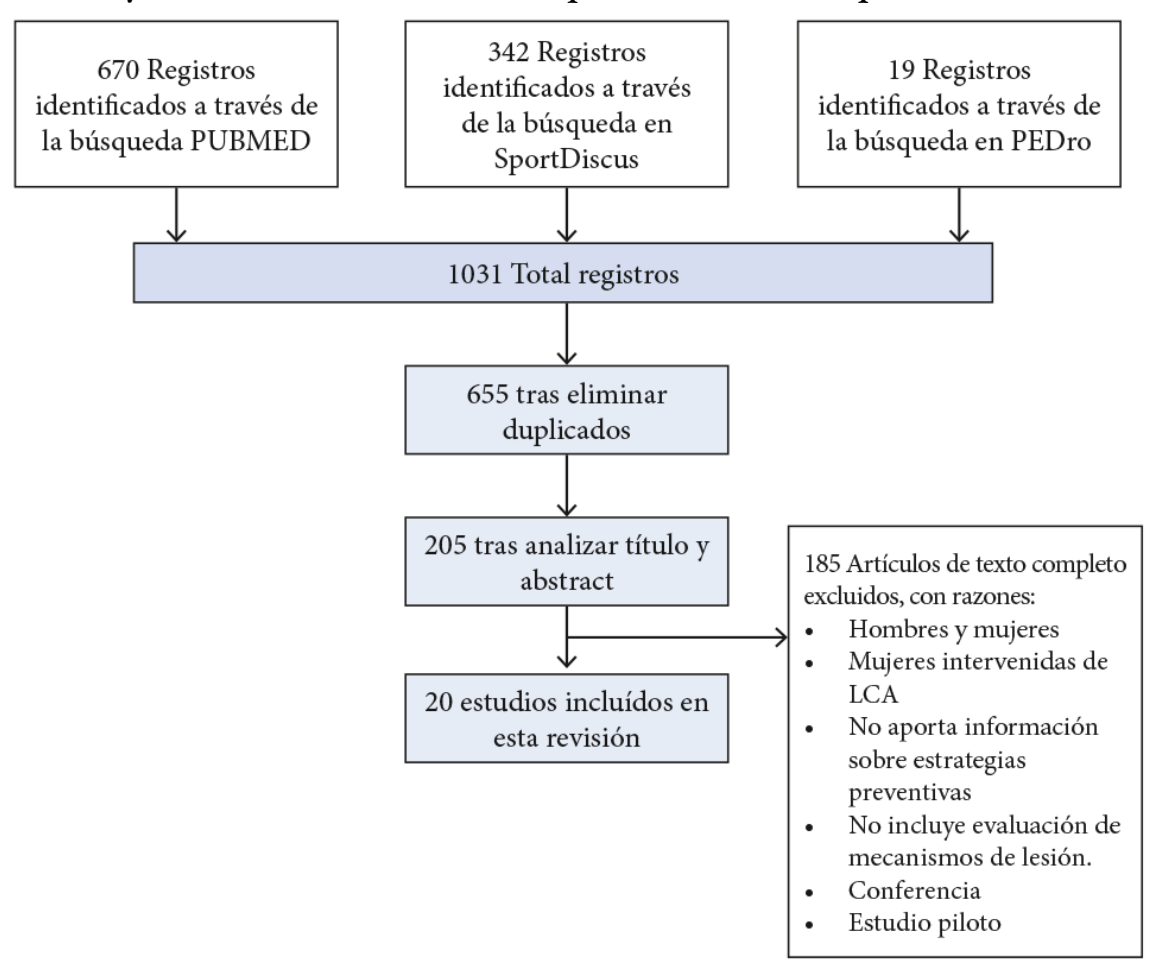

FIGURA 2.

Diagrama de flujo para el análisis de datos 
$\mathrm{Al}$ tratarse de una revisión narrativa, tras la lectura y el análisis de los trabajos se puede concretar que los principales factores de riesgo responsables en las lesiones de LCA-SC son: i) bajos niveles de fuerza en la cadena posterior y en los miembros inferiores (MMII), ii) la técnica pobre en patrones de aterrizaje, iii) las asimetrías en los patrones de fuerza y movimiento, iv) bajos niveles de fuerza y estabilidad de la zona central.

De los estudios consultados se puede extraer información para el diseño de un programa PNM basado en los principales aspectos influyentes: a) respecto al fortalecimiento muscular (tabla 1 ); b) respecto a la técnica de aterrizaje y la retroalimentación (tabla 2); c) respecto a la dosis, tiempo y adherencia al entrenamiento (tabla 3 ) yd) respecto a la influencia del sexo (tabla 4).

\section{TABLA 1}

Información sobre los estudios analizados respecto a la planificación de un programa PNM de LCA-SC en relación con el fortalecimiento muscular.

\section{RESPECTO AL FORTALECIMIENTO MUSCULAR}

\begin{tabular}{ll}
\hline Voskanian (2013) & El entrenamiento de fuerza debe incluir especialmente el fortalecimiento y \\
& reclutamiento de los isquiotibiales, de los abductores de cadera y el \\
& fortalecimiento de los glúteos. Los ejercicios pliométricos deben incorporar \\
& ejercicios de agilidad de alta intensidad, trabajando en el juego de pies y en \\
& los movimientos explosivos rápidos. Hay que enfatizar en la velocidad \\
& junto con el reclutamiento muscular y la mecánica adecuada de manera \\
& creciente.
\end{tabular}

Myer et al. (2008)

Myer et al. (2005)

Steffen et al. (2008)

Welling et al. (2019)

McConnell (2002)
El trabajo de fuerza en la abducción de cadera puede mejorar la capacidad de las atletas femeninas sobre el control de la alineación de las extremidades inferiores.

El fortalecimiento del miembro inferior se debe realizar en el rango de movimiento articular completo para corregir los bajos niveles de fuerza de los isquiotibiales comunes en las atletas femeninas.

El entrenamiento se debe basar en la estabilidad central, el equilibrio, la estabilización dinámica (pliometría) y el fortalecimiento de los isquiotibiales de manera excéntrica.

El entrenamiento de fuerza progresivo debe basarse en: fase de activación de cuádriceps, fase de entrenamiento de resistencia, trabajo simétrico y fuerza muscular.

En el programa de entrenamiento es importante el trabajo del vasto interno oblicuo y el control motor de los glúteos. 
MHSALUD, ISSN: 1659-097X, 18(2), JuLIo-Diciembre, 2021, PP 1-16

Gómez-Tomás, Rial Rebullido, Chulvi-Medrano

TABLA 2

Información sobre los estudios analizados respecto a la planificación de un programa PNM de LCA-SC en relación con la técnica de aterrizaje y la retroalimentación aportada

\section{RESPECTO A LA TÉCNICA DE ATERRIZAJE Y LA RETROALIMENTACIÓN}

Popovic et al. (2018) La retroalimentación implícita induce un cambio positivo en la mecánica de aterrizaje, mientras que la retroalimentación explícita muestra la tendencia opuesta.

Benjaminse et al. (2010) La retroalimentación visual individual en la optimización del rendimiento deportivo parece ser un método de intervención efectivo en la prevención de lesiones de LCA; esto podría aplicarse a poblaciones más grandes que participan en deportes de equipo con un alto riesgo de sufrir una lesión del LCA.

Taylor et al. (2017)

Khuu et al. (2015)

Quienes juegan baloncesto pueden necesitar una mayor dedicación a las estrategias destinadas a suavizar las fuerzas en el aterrizaje y limitar la tensión del LCA cuando aterrizan ángulos de flexión de rodilla poco profundos.

Las instrucciones verbales deben controlarse y/o documentarse claramente cuando se usa el salto vertical para evaluar el potencial de rendimiento atlético y el riesgo de lesiones. Además, a las personas profesionales que diseñan estrategias de mejora del rendimiento y prevención de lesiones basadas en evaluaciones del salto vertical se les recomienda considerar que el "entrenamiento" o la "indicación" durante la ejecución de la tarea podrían impactar las conclusiones extraídas. 
MHSALUD, ISSN: 1659-097X, 18(2), Julio-DiCIEMBRE, 2021, PP 1-16

Gómez-Tomás, Rial Rebullido, Chulvi-Medrano

TABLA 3

Información sobre los estudios analizados respecto a la planificación de un programa PNM de LCA-SC en relación con la dosis, tiempo y adherencia al entrenamiento

RESPECTO A DOSIS, TIEMPO Y ADHERENCIA AL ENTRENAMIENTO

$\begin{array}{ll}\text { Sugimoto et al. (2017) } & \text { Las personas atletas independientemente del nivel escolar o del deporte } \\ & \text { tienen altos índices (casi el 90\%) de compromiso y de aplicabilidad en las } \\ & \text { intervenciones destinadas a la prevención de lesiones. }\end{array}$

LaBella et al. (2011)

Tiempos de calentamiento de 20 minutos o menos no resultaron estadísticamente significativos, pero los equipos que utilizaron el calentamiento con mayor frecuencia tuvieron tasas más bajas de lesiones de la extremidad.

Nessler et al. (2017)

La incorporación del entrenamiento neuromuscular con fortalecimiento a una edad más temprana es beneficiosa para la prevención de lesiones. El tiempo dedicado al programa de prevención aún no se ha estandarizado para obtener los resultados más efectivos. Actualmente la mayoría de los estudios coinciden en que se necesitan sesiones de 20-30 minutos varias veces por semana.

Michaelidis
Koumantakis (2014)

\& Un programa de entrenamiento exitoso para la prevención de lesiones de LCA debe comenzar en la pretemporada durante al menos 6 semanas y continuar en temporada con menos frecuencia ( $1 / 2$ veces por semana). Los componentes del entrenamiento deben ser: el entrenamiento de fuerza, la pliometría junto con el equilibrio, la propiocepción y la retroalimentación educativa. 
TABLA 4

Información sobre los estudios analizados respecto a la planificación de un programa PNM de LCA-SC en relación con la influencia del sexo

\section{RESPECTO AL SEXO (Hombre-Mujer)}

\begin{tabular}{ll}
\hline Arundale et al. (2018) & Los resultados sugieren que las mujeres pueden requerir un mayor \\
& fortalecimiento del miembro inferior, en concreto del cuádriceps, para \\
& mejorar el riesgo de una nueva lesión.
\end{tabular}

Pallavi et al. (2017)

Los sujetos mostraron una mejor fuerza durante la fase folicular seguida de una menor fuerza durante la fase lútea, que se deterioró aún más durante la fase de la menstruación.

Zazulak et al. (2005)

La estabilidad central es el factor relacionado en el riesgo de lesiones de rodilla, ligamento y LCA con alta sensibilidad y especificidad moderada en mujeres.

Pollard et al. (2009)

Tras el salto, los momentos con menor flexión de rodilla y cadera tienen mayor ángulo de valgo de rodilla. Este perfil biomecánico puede poner a las mujeres en mayor riesgo de lesión del ligamento cruzado anterior. Las mujeres realizan maniobras atléticas con disminución de la flexión de la rodilla.

Landry et al. (2007)

Se encontraron diferencias en las formas de onda cinemáticas, cinéticas y de activación muscular entre géneros. Las mujeres presentaron el gastrocnemio lateral, gastrocnemio medial y recto femoral más activos y también demostraron un menor ángulo de flexión de cadera en comparación con los hombres durante la fase de apoyo. Estos aspectos son responsables de mayor tasa de lesiones de LCA-SC en las mujeres atletas.

Tras el análisis los artículos, se pueden establecer cuatro grupos de intervención mediante el programa de entrenamiento neuromuscular que resulta fundamental para planificar un programa la prevención de lesiones de LCA-SC (ver figura 3).

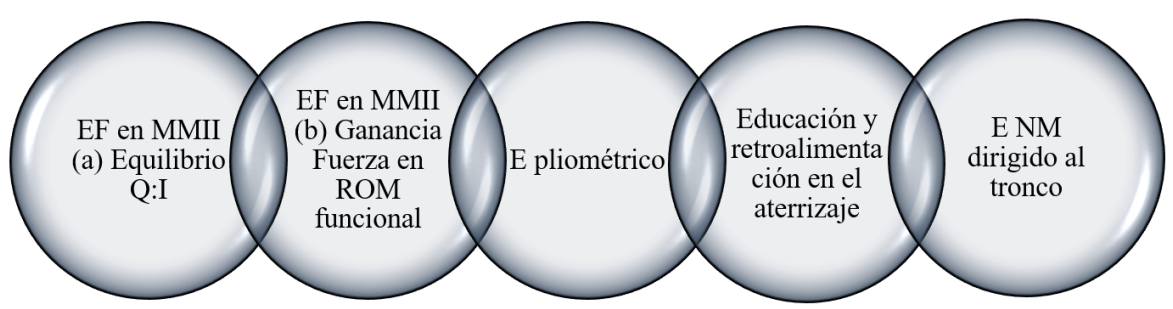

FIGURA 3.

Ilustración de la visión integradora de un programa PNM de lesiones del LCA-SC.

${ }^{*} \mathrm{EF}$ : entrenamiento de fuerza; Q:I: Ratio cuádriceps : isquiotibiales; ROM: rango de movimiento; NM: neuromuscular.

Adicionalmente, se deben considerar los efectos que puede ejercer el ciclo menstrual de la mujer; sin embargo, los datos resultan contradictorios sobre qué momento del ciclo resulta más proclive a incrementar el riesgo de lesión debido a las influencias hormonales (Hewett et al., 2007; Morse et al., 2013; Tabar, 2014). Ante esta situación, se debe subrayar la importancia de ajustar el entrenamiento a las diferentes subetapas del ciclo menstrual atendiendo a unas consideraciones generales. El ciclo menstrual representa al factor de riesgo hormonal de LCA-SC en las mujeres jugadoras de baloncesto. Esta relación entre el riesgo de lesión 
y sistema hormonal estriba en las oscilaciones de las hormonas femeninas que gobiernan el ciclo menstrual. El estradiol, la progesterona y la relaxina son las hormonas predominantes estudiadas en el ciclo menstrual y relacionadas con la laxitud del LCA. El estradiol y la progesterona se encuentran en sus niveles más bajos durante la menstruación al comienzo del ciclo menstrual (días 1-6). El estradiol alcanza su concentración máxima alrededor del momento de la ovulación (días 12-14), con un segundo aumento más bajo en la fase lútea (días 20-24). La progesterona comienza un aumento gradual en la fase folicular tardía justo antes de la ovulación, pero sus niveles más altos se alcanzan en la fase lútea media (días 19-24). Estas fluctuaciones hormonales periódicas en el ciclo menstrual causan laxitud de los ligamentos, lo que aumenta el riesgo de lesiones del LCA. En concreto, la exposición del LCA al estradiol da como resultado una reducción en la síntesis de fibroblastos y colágeno (Herzberg et al., 2017). La relaxina contribuye a la laxitud de la sínfisis púbica durante el embarazo y el parto y, de manera similar, ejerce efecto en el LCA en mujeres no embarazadas (Dragoo et al., 2011; Faryniarz et al., 2006).

\section{Discusión}

El objetivo principal de esta revisión narrativa fue detectar y analizar las evidencias existentes en relación a los programas de PNM para la lesión de LCA-SC en jugadoras de baloncesto y proponer un programa de PNM específico. Tras la lectura y el análisis detallado de los estudios, los principales hallazgos del análisis resultante en relación con los factores de riesgo encontrados son: i) Bajos niveles de fuerza en la cadena posterior y en los miembros inferiores (MMII); ii) La técnica pobre en patrones de aterrizaje; iii) Las asimetrías en los patrones de fuerza y movimiento y iv) Los bajos niveles de fuerza y estabilidad de la zona central. En segundo lugar, los aspectos fundamentales a tener en cuenta en la planificación de un programa de PNM deben incluir: i) Fortalecimiento de MMII; ii) Pliometría de miembros inferiores; iii) Técnicas de aterrizaje y retroalimentación; iv) Trabajo de estabilidad central. Estos aspectos serán discutidos a continuación.

Previo al desarrollo de los factores de riesgo encontrados, así como los criterios de prevención basados en los mismos, resulta conveniente incidir en la importancia que posee el calentamiento (Jeffreys, 2018; La Bella et al., 2011). Particularmente el calentamiento debería incluir inicialmente una parte de elevación de temperatura seguida de movilidad dinámica a nivel articular, tratando de incluir movimientos específicos deportivos; en tercer lugar, añadir ejercicios de propiocepción, y finalmente se sugiere incluir ejercicios para la potenciación neuromuscular (Olsen et al., 2005; Jeffreys, 2018).

En relación con el programa PNM de lesiones de LCA-SC en jugadoras de baloncesto, se debe atender en primer lugar al entrenamiento de fuerza en MMII. Las mujeres tienen un menor desarrollo en la musculatura isquiosural y glútea que los hombres (Zazulak et al., 2007) y además un mayor riesgo de sufrir la "posición de no retorno" (los abductores y extensores de la cadera se bloquean y la pelvis y la cadera pierden el control, produciéndose una rotación interna. La rodilla se encuentra en valgo y sufre una rotación tibial externa) (Ireland, 2002). El aumento de la activación del cuádriceps se ha asociado con un aumento de las fuerzas de deslizamiento anterior de la tibia, desencadenando una fuerza de cizalla que incrementa la tensión sobre el LCA (Brown et al., 2009; Shultz et al., 2009). En 2004 se encontró que las atletas demostraron una disminución significativa de la abducción femoral y la fuerza de rotación externa así como de resistencia del cuadrado lumbar en comparación con los atletas masculinos (Leetun et al., 2004).

Los programas que incorporaron el entrenamiento de fuerza fueron los más efectivos para disminuir las tasas de lesiones de LCA-SC. Sin embargo, el entrenamiento de fuerza por sí solo puede no ser eficaz para la prevención (Nessler et al., 2017). El entrenamiento de fuerza debe incluir especialmente el fortalecimiento de los isquiosurales, de los abductores de cadera (ayudar a reducir el valgo de la rodilla), del glúteo y de la zona central. Además, se debe abordar cualquier asimetría en los patrones de fuerza y movimiento (Voskanian, 2013), como pueda ser una debilidad marcada en la fuerza del glúteo medio y trabajar con flexión de cadera para involucrar al recto femoral (Mangine et al., 2018). 
El Colegio Americano de Medicina del Deporte (ACSM) recomienda que el entrenamiento de fuerza se realice con una frecuencia de dos a tres veces por semana. Se deben realizar entre dos o cuatro series de ejercicios (8-12 repeticiones) al 60\%-80\% (intensidad moderada a fuerte) de una repetición de máximo esfuerzo (1RM). El tiempo de descanso entre ejercicios debe ser de 2-3 min con la finalidad de recuperar la hipertrofia muscular y la fuerza en individuos sanos. Si se tiene en cuenta la frecuencia, el número de repeticiones, el ejercicio unilateral y bilateral junto con la variabilidad de los ejercicios dentro de un mismo entrenamiento, se puede conseguir un entrenamiento de fuerza de manera progresiva y una mejora del rendimiento físico del deportista (Garber et al., 2011).

El segundo aspecto a tener en cuenta son los ejercicios pliométricos. Se deben incorporar ejercicios de alta intensidad que trabajen la agilidad de pies junto con movimientos rápidos y explosivos que enfaticen la potencia y la velocidad con una dificultad creciente. Se pueden incorporar saltos, cortes y movimientos laterales con "perturbación" o desequilibrios para ver si el atleta mantiene la forma adecuada de trabajo. Estos tipos de entrenamiento propioceptivo y de equilibrio pueden mejorar el control postural (Arundale et al., 2018; Nessler et al., 2017; Sugimoto et al., 2014) y hacer que el entrenamiento empiece a parecerse más a una situación deportiva real (Voskanian, 2013).

El tercer aspecto importante en el programa PNM de lesiones LCS-SC es la educación y la retroalimentación en el aterrizaje. La instrucción de estímulos verbales, visuales, de video o retroalimentación de manera implícita mediante una proyección visual pueden facilitar el aprendizaje del gesto motor (Gilchrist et al., 2008; Olsen et al., 2005; Popovic et al., 2018; Steffen et al., 2008). Este factor resulta muy importante debido a la elevada complicación de la tarea, que adicionalmente se incrementa por los factores intrínsecos de la mujer (p.ej. mayor ángulo Q, predominancia de activación de cuádriceps frente a isquiosural, etc.)(McCormick, 2012). Un aterrizaje con la rodilla cerca a la extensión o con un ángulo menor a $45^{\circ}$ de flexión de rodilla hace que el cuádriceps actúe de antagonista del LCA y empuje la tibia hacia anterior aumentando la tensión en el ligamento (Boden et al., 2000). Los hombres, ante un estímulo visual, mostraron mayor momento de flexión y de fuerza de reacción de la rodilla en los cortes y deslizamientos laterales que las mujeres, posiblemente debido a que aterrizan con dominancia del LCA (movimiento medial de la rodilla, valgo y alto momento de la fuerza de reacción) (Benjaminse et al., 2017).

Finalmente, el cuarto aspecto a tener en cuenta es el entrenamiento de estabilidad central dirigido al tronco. La estabilización central del cuerpo es especialmente necesaria para evitar lesiones secundarias por falta de control corporal de la extremidad inferior. Además, en las mujeres la relación existente entre estabilización central y riesgo de lesión de rodilla es estrecha (Zazulak et al., 2007). Los estabilizadores del tronco y de la cadera deben preactivarse para contrarrestar el movimiento del tronco y regular las posturas de las extremidades inferiores (Myer et al., 2008) aumentando el control de la aducción y rotación interna durante las actividades funcionales de carga, y disminuir la tendencia hacia el valgo (Zazulak et al., 2005).

Hay que añadir la importancia del factor hormonal en las mujeres. Se considera que el $41.7 \%$ de las mujeres que realizan ejercicio físico manifiestan que su ciclo menstrual tiene un impacto negativo en el entrenamiento y su rendimiento deportivo (Bruinvels et al., 2017). A lo largo de las fases del ciclo menstrual las fluctuaciones en los niveles hormonales de esteroides femeninos afectan el sistema nervioso autónomo y las funciones metabólicas, modificando algunos de los parámetros fisiológicos que pueden afectar al rendimiento físico (la fuerza, la potencia, la resistencia anaeróbica e incluso la velocidad de inicio en el entrenamiento (Wiecek et al., 2016) y psicológico (Pallavi et al., 2017). Del mismo modo, las variaciones en las concentraciones de estrógenos y progesterona pueden provocar cambios en la estructura y función del colágeno, lo que consecuentemente influye sobre las propiedades mecánicas del tejido conectivo así como en la laxitud tendinosa (Morse et al., 2013), la laxitud ligamentosa y la propensión a la lesión del ligamento cruzado anterior (Tabar, 2014). Las atletas femeninas pueden estar más predispuestas a las lesiones de LCASC durante la fase preovulatoria del ciclo menstrual (Hewett et al., 2007). Pero el mismo año también se encontró una mayor laxitud durante las fases ovulatoria y post-ovulatoria (Zazulak et al., 2007). Existe 
evidencia preliminar que sugiere que la laxitud de la rodilla ligamentosa cambia a lo largo del ciclo menstrual de la mujer, y la mayoría de los estudios informan que el mayor cambio se produce durante la fase ovulatoria. Sin embargo, es importante señalar que la evidencia sigue siendo inconsistente (Belanger et al., 2013).

Las recomendaciones más extendidas son la inclusión de todos los factores expuestos anteriormente dentro de los programas de prevención, con el objetivo de lograr una sinergia que estabilice la rodilla y proteja el LCA.

A modo de consideraciones finales, es importante remarcar que el entrenamiento neuromuscular se debe realizar preferiblemente con una duración superior a 20 minutos por sesión, y se debe llevar a cabo tanto antes como durante la temporada. Del mismo modo se debe implementar antes o durante el inicio de la pubertad para evitar que se desarrollen patrones neuromusculares y biomecánicos incorrectos (Sugimoto et al., 2014). Empezar con la intervención lo más temprano posible, entre 6-10 años, ofrece la posibilidad de poder sugerir un continuum en la programación de los entrenes y la prevención de las lesiones (Renstrom et al., 2008). El entrenamiento neuromuscular junto con el entrenamiento de fuerza a una edad temprana son la clave para reducir las lesiones de LCA-SC (Nessler et al., 2017). Resulta muy importante entender este punto de vista, dado que a veces la mujer tiene problemas para mejorar sus factores intrínsecos.

Finalmente se considera que un continuum es necesario para crear una alfabetización motora, la cual incrementa el rendimiento motor tanto como reduce el riesgo de lesión (Faigenbaum \& Rebullido, 2018).

\section{LIMITACIONES DEL ESTUDIO}

Una de las principales limitaciones que presente esta revisión narrativa es la falta de un abordaje cuantitativo de los resultados. Sin embargo, consideramos que este tipo de estudio permite contextualizar la lesión de LCA-SC en la mujer deportista, así como visualizar una propuesta actual sobre la aplicación de estrategias de prevención neuromuscular en las lesiones de LCA-SC para ser aplicadas en las mujeres. Las líneas de investigación futura deberían centrarse en la relación dosis-respuesta en la prevención de lesiones de LCA-SC en función de la edad. Finalmente, al existir limitada información específica de las jugadoras de baloncesto, se han integrado también los datos que se desprenden de artículos que incluyen mujeres jugadoras de otros deportes.

\section{Conclusiones}

En jugadoras de baloncesto la lesión de LCA-SC es frecuente, y tiene múltiples factores intrínsecos que pueden mejorarse aplicando un programa de PNM adecuado que incluya: a) Entrenamiento de fuerza progresivo para miembros inferiores orientado a incrementar la fuerza en ROM funcional y a generar equilibrio del ratio cuádriceps:isquiosurales; b) Pliometría; c) Entrenamiento del núcleo y entrenamiento neuromuscular dirigido al tronco; d) Educación y retroalimentación en los diferentes tipos de aterrizajes. Asimismo, se debe tomar en consideración la influencia hormonal que ejerce el ciclo menstrual. Por lo tanto, se propone que los programas de prevención para reducir la incidencia de lesión LCA-SC en jugadoras de baloncesto enfaticen la importancia de comenzar su aplicación tan pronto como sea posible, con la intención de crear una alfabetización motora y reducir el riesgo de lesión, así como atender a las subfases del ciclo menstrual.

\section{RECOMENDACIONES}

Es importante realizar investigaciones que: 
- Comparen las diferencias o semejanzas entre sexos, para ofrecer recomendaciones más concretas y específicas.

- Analicen el esquema motor de un niño/a entrenado acorde a sus exigencias temporales y sus implicaciones sobre la prevención de LCA-SC.

- Orienten a las personas profesionales a la hora de planificar los entrenamientos o los programas de prevención de lesiones de forma individualizada atendiendo a todas las particularidades específicas (p. ej. edad, ciclo menstrual, estatus de entrenamiento, etc.).

- Incluyan programas de prevención a largo plazo, atendiendo al continuum de edad biológica.

\section{REFERENCIAS}

Aerts, I., Cumps, E., Verhagen, E., Wuyts, B., Van De Gucht, S., \& Meeusen, R. (2015). The effect of a 3-month prevention program on the jump-landing technique in basketball: a randomized controlled trial.J Sport Rehabil, 24(1), 21-30. https://doi.org/10.1123/jsr.2013-0099

Ahmad, C. S., Clark, A. M., Heilmann, N., Schoeb, J. S., Gardner, T. R., \& Levine, W. N. (2006). Effect of gender and maturity on quadriceps-to-hamstring strength ratio and anterior cruciate ligament laxity. Am J Sports Med, 34(3), 370-374. https://doi.org/10.1177/0363546505280426

Arundale, A. J. H., Capin, J. J., Zarzycki, R., Smith, A., \& Snyder-Mackler, L. (2018). Functional and patient-reported outcomes improve over the course of rehabilitation: A secondary analysis of the ACL-SPORTS trial. Sports Health, 10(5), 441-452. https://doi.org/10.1177/1941738118779023

Belanger, L., Burt, D., Callaghan, J., Clifton, S., \& Gleberzon, B. J. (2013). Anterior cruciate ligament laxity related to the menstrual cycle: an updated systematic review of the literature. J Can Chiropr Assoc, 57(1), 76-86. PMID: 23483028.

Benjaminse, A., Lemmink, K. A., Diercks, R. L., \& Otten, B. (2010). An investigation of motor learning during sidestep cutting: design of a randomised controlled trial. BMC Musculoskelet Disord, 11, 235. https://doi.org/10.1 186/1471-2474-11-235

Benjaminse, A., Otten, B., Gokeler, A., Diercks, R. L., \& Lemmink, K. (2017). Motor learning strategies in basketball players and its implications for ACL injury prevention: a randomized controlled trial. Knee Surg Sports Traumatol Arthrosc, 25(8), 2365-2376. https://doi.org/10.1007/s00167-015-3727-0

Besier, T. F., Lloyd, D. G., Cochrane, J. L., \& Ackland, T. R. (2001). External loading of the knee joint during running and cutting maneuvers.Med Sci Sports Exerc, 33(7), 1168-1175. https://doi.org/10.1097/00005768-2001070 00-00014

Beynnon, B. D., Vacek, P. M., Newell, M. K., Tourville, T. W., Smith, H. C., Shultz, S. J., Slauterbeck, J. R., \& Johnson, R. J. (2014). The Effects of Level of Competition, Sport, and Sex on the Incidence of First-Time Noncontact Anterior Cruciate Ligament Injury. Am J Sports Med, 42(8), 1806-1812. https://doi.org/10.1177/036354651 4540862

Boden, B. P., Dean, G. S., Feagin, J. A., Jr., \& Garrett, W. E., Jr. (2000). Mechanisms of anterior cruciate ligament injury. Orthopedics, 23(6), 573-578. PMID: 10875418.

Brown, T. N., Palmieri-Smith, R. M., \& McLean, S. G. (2009). Sex and limb differences in hip and knee kinematics and kinetics during anticipated and unanticipated jump landings: implications for anterior cruciate ligament injury. Br J Sports Med, 43(13), 1049-1056. https://doi.org/10.1136/bjsm.2008.055954

Bruinvels, G., Burden, R. J., McGregor, A. J., Ackerman, K. E., Dooley, M., Richards, T., \& Pedlar, C. (2017). Sport, exercise and the menstrual cycle: where is the research?. Br J Sports Med, 51(6), 487-488. https://doi.org/10.1 136/bjsports-2016-096279

Caldemeyer, L. E., Brown, S. M., \& Mulcahey, M. K. (2020). Neuromuscular training for the prevention of ankle sprains in female athletes: a systematic review. Phys Sportsmed, 48(4), 363-369. https://doi.org/10.1080/0091 3847.2020 .1732246 
MHSAlud, ISSN: 1659-097X, 18(2), Julio-Diciembre, 2021, PP 1-16

Gómez-Tomás, Rial Rebullido, Chulvi-Medrano

Chappell, J. D., Creighton, R. A., Giuliani, C., Yu, B., \& Garrett, W. E. (2007). Kinematics and electromyography of landing preparation in vertical stop-jump: risks for noncontact anterior cruciate ligament injury. Am J Sports Med, 35(2), 235-241. https://doi.org/10.1177/0363546506294077

Cherni, Y., Jlid, M. C., Mehrez, H., Shephard, R. J., Paillard, T., Chelly, M. S., \& Hermassi, S. (2019). Eight Weeks of Plyometric Training Improves Ability to Change Direction and Dynamic Postural Control in Female Basketball Players. Front Physiol, 10, 1-10. https://doi.org/10.3389/fphys.2019.00726

Dragoo, J. L., Castillo, T. N., Braun, H. J., Ridley, B. A., Kennedy, A. C., \& Golish, S. R. (2011). Prospective correlation between serum relaxin concentration and anterior cruciate ligament tears among elite collegiate female athletes. Am J Sports Med, 39(10), 2175-2180. https://doi.org/10.1177/0363546511413378

Faigenbaum, A. D., \& Rebullido, T. R. (2018). Understanding physical literacy in youth.J Strength Cond Res, 40(6), 90-94. https://doi.org/10.1519/SSC.0000000000000417

Faryniarz, D. A., Bhargava, M., Lajam, C., Attia, E. T., \& Hannafin, J. A. (2006). Quantitation of estrogen receptors and relaxin binding in human anterior cruciate ligament fibroblasts. In Vitro Cell Dev Biol Anim, 42(7), 176-181. https://doi.org/10.1290/0512089.1

Garber, C. E., Blissmer, B., Deschenes, M. R., Franklin, B. A., Lamonte, M. J., Lee, I.-M.,Nieman, D. C., \& Swain, D. P. (2011). American College of Sports Medicine position stand. Quantity and quality of exercise for developing and maintaining cardiorespiratory, musculoskeletal, and neuromotor fitness in apparently healthy adults: guidance for prescribing exercise. Med Sci Sports Exerc, 43(7), 1334-1359. https://doi.org/10.1249/MSS.0b013e31821 $3 \mathrm{fefb}$

Gilchrist, J., Mandelbaum, B. R., Melancon, H., Ryan, G. W., Silvers, H. J., Griffin, L. Y., Watanabe, D. S., Randall, W. D., \& Dvorak, J. (2008). A randomized controlled trial to prevent noncontact anterior cruciate ligament injury in female collegiate soccer players. Am J Sports Med, 36(8), 1476-1483. https://doi.org/10.1177/03635 46508318188

Herzberg, S. D., Motu'apuaka, M. L., Lambert, W., Fu, R., Brady, J., \& Guise, J. M. (2017). The Effect of Menstrual Cycle and Contraceptives on ACL Injuries and Laxity: A Systematic Review and Meta-analysis. OrthopJ Sports Med, 5(7), 1-10. https://doi.org/10.1177/2325967117718781

Hewett, T. E., Ford, K. R., Hoogenboom, B. J., \& Myer, G. D. (2010). Understanding and preventing ACL injuries: current biomechanical and epidemiologic considerations - update 2010. N Am J Sports Phys Ther, 5(4), 234-251. PMID: 21655382.

Hewett, T. E., Zazulak, B. T., \& Myer, G. D. (2007). Effects of the menstrual cycle on anterior cruciate ligament injury risk: a systematic review. Am J Sports Med, 35(4), 659-668. https://doi.org/10.1177/0363546506295699

Ireland, M. L. (2002). The female ACL: why is it more prone to injury?. Orthop Clin North Am, 33(4), 637-651. ht tps://doi.org/10.1016/s0030-5898(02)00028-7

Jordan, M. J., Aagaard, P., \& Herzog, W. (2017). Anterior cruciate ligament injury/reinjury in alpine ski racing: a narrative review. Open Access J Sports Med, 8, 71-83. https://doi.org/10.2147/OAJSM.S106699

Jeffreys, I. (2018). The warm-up: Maximize performance and improve long-term athletic development. Human Kinetics.

Khuu, S., Musalem, L. L., \& Beach, T. A. C. (2015). Verbal instructions acutely affect drop vertical jump biomechanics implications for athletic performance and injury risk assessments. J Strength Cond Res, 29(10), 2816-2826. htt ps://doi.org/10.1519/JSC.0000000000000938

LaBella, C. R., Huxford, M. R., Grissom, J., Kim, K. Y., Peng, J., \& Christoffel, K. K. (2011). Effect of neuromuscular warm-up on injuries in female soccer and basketball athletes in urban public high schools: cluster randomized controlled trial. Arch Pediatr Adolesc Med, 165(11), 1033-1040. https://doi.org/10.1001/archpediatrics.2011 .168

Landry, S. C., McKean, K. A., Hubley-Kozey, C. L., Stanish, W. D., \& Deluzio, K. J. (2007). Neuromuscular and lower limb biomechanical differences exist between male and female elite adolescent soccer players during an unanticipated side-cut maneuver. Am J Sports Med, 35(11), 1888-1900. https://doi.org/10.1177/0363546507 300823 
Leetun, D. T., Ireland, M. L., Willson, J. D., Ballantyne, B. T., \& Davis, I. M. (2004). Core stability measures as risk factors for lower extremity injury in athletes. Med Sci Sports Exerc, 36(6), 926-934. https://doi.org/10.1249/0 1.mss.0000128145.75199.c3

Mangine, G. T., Redd, M. J., Gonzalez, A. M., Townsend, J. R., Wells, A. J., Jajtner, A. R., Beyer, K.S., Boone, C.H., La Monica, M.B., Stout, J. R., Fukuda, D.H., Ratamess, N.A., \& Hoffman, J.R. (2018). Resistance training does not induce uniform adaptations to quadriceps. PLoS One, 13(8). https://doi.org/10.1371/journal.pone.0198304

McConnell, J. (2002). The physical therapist's approach to patellofemoral disorders. Clin Sports Med, 21(3), 363-387. https://doi.org/10.1016/s0278-5919(02)00027-3

McCormick, B. T. (2012). Task complexity and jump landings in injury prevention for basketball players. Strength Cond J, 34(2), 89. https://doi.org/10.1519/SSC.0b013e31823ee08e

Michaelidis, M., \& Koumantakis, G. A. (2014). Effects of knee injury primary prevention programs on anterior cruciate ligament injury rates in female athletes in different sports: A systematic review. Phys Ther Sport, 15(3), 200-210. https://doi.org/10.1016/j.ptsp.2013.12.002

Morse, C. I., Spencer, J., Hussain, A. W., \& Onambele, G. L. (2013). The effect of the oral contraceptive pill on the passive stiffness of the human gastrocnemius muscle in vivo. J Musculoskelet Neuronal Interact, 13(1), 97-104. PMID: 23445919.

Munro, A., Herrington, L., \& Comfort, P. (2012). Comparison of landing knee valgus angle between female basketball and football athletes: Possible implications for anterior cruciate ligament and patellofemoral joint injury rates. Phys Ther Sport, 13(4), 259-264. https://doi.org/10.1016/j.ptsp.2012.01.005

Myer, G. D., Chu, D. A., Brent, J. L., \& Hewett, T. E. (2008). Trunk and hip control neuromuscular training for the prevention of knee joint injury. Clin Sports Med, 27(3), 425-448. https://doi.org/10.1016/j.csm.2008.02.006

Myer, G. D., Ford, K. R., Palumbo, J. P., \& Hewett, T. E. (2005). Neuromuscular training improves performance and lower extremity biomechanics in female athletes. J Strength Cond Res, 19(1), 51-60. https://doi.org/10.1519/ 13643.1

Nessler, T., Denney, L., \& Sampley, J. (2017). ACL Injury Prevention: What Does Research Tell Us?. Curr Rev Musculoskelet Med, 10(3), 281-288. https://doi.org/10.1007/s12178-017-9416-5

Olsen, O. E., Myklebust, G., Engebretsen, L., Holme, I., \& Bahr, R. (2005). Exercises to prevent lower limb injuries in youth sports: cluster randomised controlled trial. BMJ, 330(7489), 1-7. https://doi.org/10.1136/bmj.3833 $0.632801 .8 \mathrm{~F}$

Omi, Y., Sugimoto, D., Kuriyama, S., Kurihara, T., Miyamoto, K., Yun, S., Kawashima, T., \& Hirose, N. (2018). Effect of Hip-Focused Injury Prevention Training for Anterior Cruciate Ligament Injury Reduction in Female Basketball Players: A 12-Year Prospective Intervention Study. Am J Sports Med, 46(4), 852-861. https://doi.o $\mathrm{rg} / 10.1177 / 0363546517749474$

Pallavi, L., Souza, U. J. D., \& Shivaprakash, G. (2017). Assessment of musculoskeletal strength and levels of fatigue during different phases of menstrual cycle in young adults. J Clin Diagn Res, 11(2), CC11. https://doi.org/10 $.7860 / J C D R / 2017 / 24316.9408$

Pollard, C. D., Sigward, S. M., \& Powers, C. M. (2009). Limited hip and knee flexion during landing is associated with increased frontal plane knee motion and moments. Clin Biomech (Bristol, Avon), 25(2), 142-146. https://doi. org/10.1016/j.clinbiomech.2009.10.005

Popovic, T., Caswell, S. V., Benjaminse, A., Siragy, T., Ambegaonkar, J., \& Cortes, N. (2018). Implicit video feedback produces positive changes in landing mechanics. J Exp Orthop, 5(1), 12. https://doi.org/10.1186/s40634-018 $-0129-5$

Renstrom, P., Ljungqvist, A., Arendt, E., Beynnon, B., Fukubayashi, T., Garrett, W., W., Georgoulis, W., Hewett, T. E., Johnson, R., Krosshaug, T., Mandelabaum, B., Micheli, L., Myklebust, G., Roos, E., Roos, H., Schamasch, P., Schultz, S., Werner, S., Wojtys, E., \& Engebretsen, L. (2008). Non-contact ACL injuries in female athletes: an International Olympic Committee current concepts statement. Br J Sports Med, 42(6), 394-412. https://doi.o $\mathrm{rg} / 10.1136 / \mathrm{bjsm} .2008 .048934$ 
MHSALUD, ISSN: 1659-097X, 18(2), JULIO-DiCIEMBRE, 2021, PP 1-16

Gómez-Tomás, Rial Rebullido, Chulvi-Medrano

Riva, D., Bianchi, R., Rocca, F., \& Mamo, C. (2016). Proprioceptive training and injury prevention in a professional men's basketball team: a six-year prospective study.J Strength Cond Res, 30(2), 461. https://doi.org/10.1519/ JSC.0000000000001097

Roos, K. G., Marshall, S. W., Kerr, Z. Y., Golightly, Y. M., Kucera, K. L., Myers, J. B., Rosamond, W.D., \& Comstock, R. D. (2015). Epidemiology of Overuse Injuries in Collegiate and High School Athletics in the United States. Am J Sports Med, 43(7), 1790-1797. https://doi.org/10.1177/0363546515580790

Sanders, T. L., Maradit Kremers, H., Bryan, A. J., Larson, D. R., Dahm, D. L., Levy, B. A., Stuart, M. J., \& Krych, A. J. (2016). Incidence of Anterior Cruciate Ligament Tears and Reconstruction: A 21-Year Population-Based Study. Am J Sports Med, 44(6), 1502-1507. https://doi.org/10.1177/0363546516629944

Shultz, S. J., Nguyen, A. D., Leonard, M. D., \& Schmitz, R. J. (2009). Thigh strength and activation as predictors of knee biomechanics during a drop jump task. Med Sci Sports Exerc, 41(4), 857-866. https://doi.org/10.1249/M SS.0b013e3181e3b3f

Steffen, K., Myklebust, G., Olsen, O. E., Holme, I., \& Bahr, R. (2008). Preventing injuries in female youth football --a cluster-randomized controlled trial. Scand J Med Sci Sports, 18(5), 605-614. https://doi.org/10.1111/j.160 0-0838.2007.00703.x

Sugimoto, D., Mattacola, C. G., Bush, H. M., Thomas, S. M., Foss, K. D., Myer, G. D., \& Hewett, T. E. (2017). Preventive Neuromuscular Training for Young Female Athletes: Comparison of Coach and Athlete Compliance Rates.J Athl Train, 52(1), 58-64. https://doi.org/10.4085/1062-6050-51.12.20

Sugimoto, D., Myer, G. D., Foss, K. D., \& Hewett, T. E. (2014). Dosage effects of neuromuscular training intervention to reduce anterior cruciate ligament injuries in female athletes: meta- and sub-group analyses. Sports Med, 44(4), 551-562. https://doi.org/10.1007/s40279-013-0135-9

Tabar, V. (2014). Revisión: influencia de los cambios hormonales endógenos sobre las lesiones y la laxitud del ligamento cruzado anterior en mujeres deportistas. (Tesis de grado) Universidad Politécnica de Navarra, Pamplona. https://academica-e.unavarra.es/bitstream/handle/2454/12023/TFGVirginiaTabarLusarreta.pdf? sequence $=1 \&$ is Allowed $=\mathrm{y}$

Taylor, J. B., Ford, K. R., Schmitz, R. J., Ross, S. E., Ackerman, T. A., \& Shultz, S. J. (2017). Biomechanical differences of multidirectional jump landings among female basketball and soccer players. J Strength Cond Res, 31(11), 3034-3045. https://doi.org/10.1519/JSC.0000000000001785

Voskanian, N. (2013). ACL Injury prevention in female athletes: review of the literature and practical considerations in implementing an ACL prevention program. Curr Rev Musculoskelet Med, 6(2), 158-163. https://doi.org/1 0.1007/s12178-013-9158-y

Welling, W., Benjaminse, A., Lemmink, K., Dingenen, B., \& Gokeler, A. (2019). Progressive strength training restores quadriceps and hamstring muscle strength within 7 months after ACL reconstruction in amateur male soccer players. Phys Ther Sport, 40, 10-18. https://doi.org/10.1016/j.ptsp.2019.08.004

Wiecek, M., Szymura, J., Maciejczyk, M., Cempla, J., \& Szygula, Z. (2016). Effect of sex and menstrual cycle in women on starting speed, anaerobic endurance and muscle power. Physiol Int, 103(1), 127-132. https://doi.org/10.15 56/036.103.2016.1.13

Zazulak, B. T., Hewett, T. E., Reeves, N. P., Goldberg, B., \& Cholewicki, J. (2007). Deficits in neuromuscular control of the trunk predict knee injury risk: a prospective biomechanical-epidemiologic study. Am J Sports Med, 35(7), 1123-1130. https://doi.org/10.1177/0363546507301585

Zazulak, B. T., Ponce, P. L., Straub, S. J., Medvecky, M. J., Avedisian, L., \& Hewett, T. E. (2005). Gender comparison of hip muscle activity during single-leg landing.J Orthop Sports Phys Ther, 35(5), 292-299. https://doi.org/10. 2519/jospt.2005.35.5.292

Zuckerman, S. L., Wegner, A. M., Roos, K. G., Djoko, A., Dompier, T. P., \& Kerr, Z. Y. (2018). Injuries sustained in National Collegiate Athletic Association men's and women's basketball, 2009/2010-2014/2015. Br J Sports Med, 52(4), 261-268. https://doi.org/10.1136/bjsports-2016-096005 